\title{
15 years of high precision astrometry in the Galactic Center
}

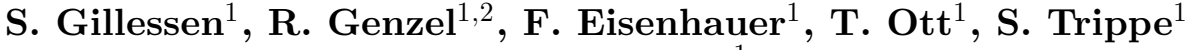 \\ and F. Martins ${ }^{1}$ \\ ${ }^{1}$ Max-Planck-Institute for extraterrestial physics \\ email: ste@mpe.mpg.de \\ ${ }^{2}$ Physics Department, University of California, Berkeley, CA 94720, USA
}

\begin{abstract}
In 1992, we obtained the first observations of S2 a star close to the supermassive black hole at the Galactic Center. In 2002, S2 passed its periastron and in 2007, it completed a first fully observed revolution. This orbit allowed us to determine the mass of and the distance to the supermassive black hole with unprecedented accuracy. Here we present a re-analysis of the data set, enhancing the astrometric accuracy to 0.5 mas and increasing the number of welldetermined stellar orbits to roughly 15 . This allows to constrain the extended mass distribution around the massive black hole and will lead in the near future to the detection of post-Newtonian effects. We will also give an outlook on the potential of interferometric near-infrared astrometry with 10 microarcsecond accuracy from the VLTI.
\end{abstract}

Keywords. Galaxy: center, astrometry, infrared: stars, instrumentation: interferometers

\section{Introduction}

The Galactic Center (GC) is a stunning example of the power of high precision astrometry. Progress in GC research has been driven by technology, in particular by high resolution techniques in the near infrared (NIR). Only the NIR allows to overcome the interstellar extinction towards the GC at intrinsically high resolution and only with sufficient angular resolution it is possible to beat the confusion of the dense stellar field. The main concern with respect to resolution from an observer's point of view is Earth's atmosphere, which implies the use of special techniques. In 1992 our group obtained the first Speckle observation of the innermost region of our Galaxy, using the custom-built camera SHARP on ESO's NTT, a 3.6m telescope. In 2002 we started using adaptive optics (AO) with the system NACO on ESO's VLT 8.2m telescope.

The most exciting result of these observations is the detection of individual Keplerian stellar orbits (Schödel et al. 2002, Ghez et al. 2005, Eisenhauer et al. 2005), allowing to probe the gravitational potential in which the so-called S-stars move. The data are consistent with a point mass of $4 \times 10^{6}$ solar masses. Spatially it coincides with the compact non-thermal radio source Sgr A* and a spurious NIR counterpart. Taken together, these observations constitute the best proof for the existence of an astrophysical black hole.

We have determined 15 full 3D stellar orbits from our data set of the central arcsecond covering 16 years. We are currently monitoring 100 stars both in astrometry as well as spectroscopy. The astrometric long-term accuracy of our AO data is $\sim 350 \mu$ as for the stars in the central arcsecond. Given the exquisit quality of the orbital data, it is natural to ask the question, whether the post-Keplerian effects can be detected.

General relativity predicts that the pericenter of the orbit of star S2 (which has the shortest orbital period of our sample and is the brightest one) should precess by $0.2^{\circ}$ per revolution of 15 years. While the Schwarzschild metric leads to a prograde precession, 


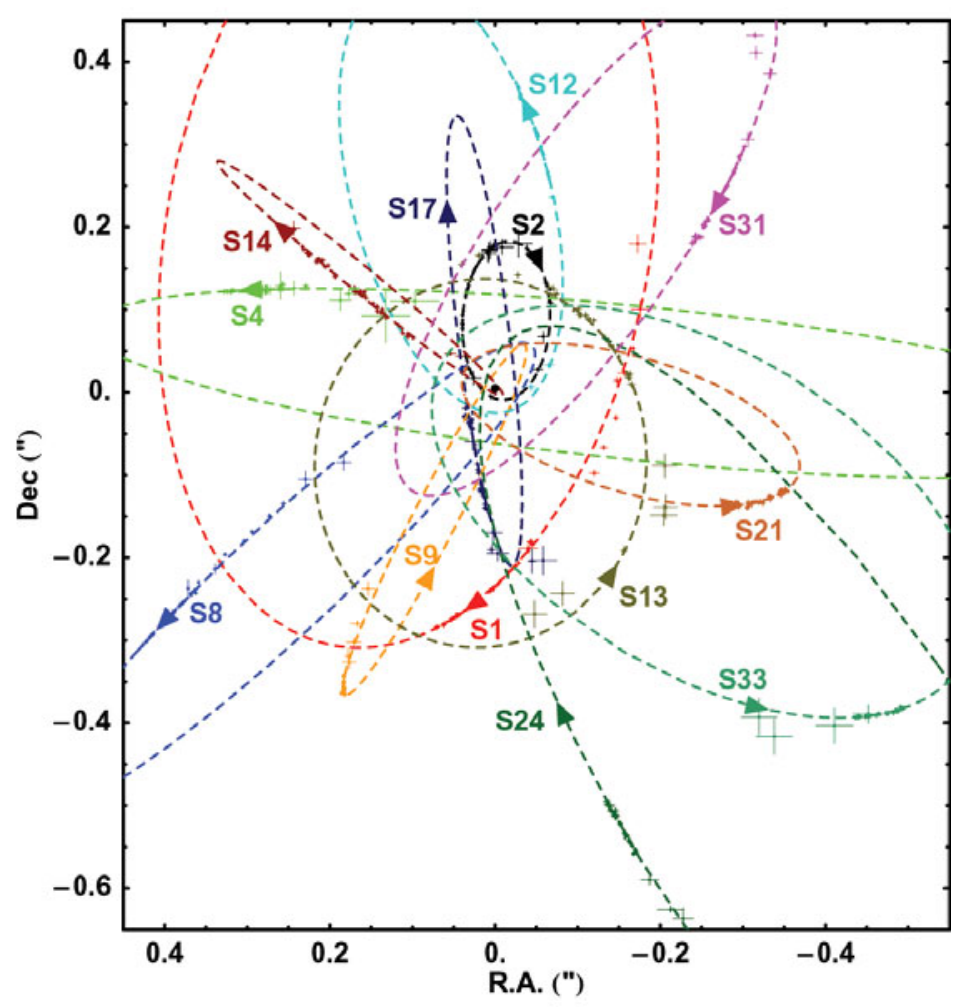

Figure 1. Stellar orbits around Sgr A*. The dashed lines are the best fitting orbits in a common Kepler potential, given the data shown as crosses. The arrows indicate the directions of motion.

there are astrophysical reasons to assume the existence of counteracting effects - a sofar unseen, extended mass component (Morris 1993, Muno et al. 2005) would lead to a retrograde precession. Since the two effects depend differently on the distance to the central mass, the system of S-stars offers the opportunity to measure both. Thus one can hope to test the Schwarzschild metric for the heaviest object for which it was ever tested and to determine the profile of a possible extended mass component.

There is a two-fold way to measure these post-Newtonian effects. First, the existing and future data from the same instruments and understanding the sources of systematic errors in the data set should allow us to unambiguously identify these effects. The second way is to increase the angular resolution further and observe objects that are even closer to the event horizon than the S-stars seen nowadays. The latter is only possible with either an increased telescope diameter or with interferometry.

\section{Astrometry of S-stars with NIR imaging}

A natural choice for the coordinate system in the GC is to put the central mass at rest at the origin of the system. Since Sgr A* is not always detectable in the NIR (either because it is in a faint state or because it is confused with stars) a convenient choice is to refer coordinates to the radio source. The cross-calibration of the two wavelength regimes can be achieved by using nine $\mathrm{SiO}$ masers for which the radio coordinates and proper motions can be measured (Reid et al. 2007). 
The accuracy with which radio Sgr A* can be unrecoved in the NIR images is limited by both the positional errors of the $\mathrm{SiO}$ masers in radio as well as the errors in their NIR positions. We have determined the error propagation by means of Monte-Carlo simulations, varying all input coordinates by their respective errors. We find that the radio source can be located to within $(1.3,1.2)$ mas in both axes. The same sort of simulations also allows to check how well the relative velocity of the two coordinate systems can be matched. We find that the velocity calibration is accurate to the level of $(0.4,0.2)$ mas $/ \mathrm{yr}$ (which corresponds to $\sim 12 \mathrm{~km} \mathrm{~s}^{-1}$ at the distance of the GC).

In contrast, the large number (60) of available epochs and high intrinsic accuracy of the NIR positions $(350 \mu \mathrm{as})$ allows to locate isolated, bright stars to $\sim 100 \mu$ as and determine their proper motions to $\sim 20 \mu \mathrm{as} / \mathrm{yr}$. This means that the positional and velocity errors with respect to Sgr A* are dominated by the uncertainty of the coordinate system. When considering the post-Newtonian effects, the velocity calibration is of particular concern. Both precession and incorrect velocity calibration can lead to non-closing orbital figures because the two effects are to some degree degenerate. Therefore, it is important to work in a proper reference frame. The current situation could be improved in a few ways:

- Future measurements of the $\mathrm{SiO}$ masers will bring the current errors down.

- The position and velocity of the coordinate system can be left free during the orbital fitting. Apparently this gives up the ability to test whether the mass coincides with radio Sgr A* and due to the degeneracy it decreases the sensitivity to post-Newtonian effects.

- At least for the velocity of the coordinate system one can get an independent calibration. In the NIR a large number of stars are seen in the central star cluster. Dynamically one expects the cluster to be a relaxed system, or being at rest with respect to the central point mass. If this assumption is correct, then one can get the velocity calibration to an accuracy of $\bar{v} / N_{\text {stars }}^{1 / 2}$ where $\bar{v}$ is the mean velocity per star.

From our data we could use 2700 stars with a mean velocity of 3.8 mas/yr. This yields an accuracy for the velocity calibration of $80 \mu \mathrm{as} / \mathrm{yr}$, considerably better than that is possible with the $\mathrm{SiO}$ masers. We have checked for the such a defined coordinate system, what an orbit fit would yield for a position and velocity of the central point mass. Essentially, both position and velocity agree with the expectations (point mass is at rest at the origin of the system) within $2 \sigma$.

Given this coordinate system, we can derive the upper limits on post-Newtonian effects. As an example, we tested a very simple model for an extended mass distribution, namely $\rho(r)=$ const. Fitting a relativistic orbit to the data of $\mathrm{S} 2$ then yields the extended mass component inside the orbit of $\mathrm{S} 2$ to be $1.7 \pm\left. 0.9\right|_{\text {stat }} \pm\left. 1.2\right|_{\text {sys }} \%$ of the central mass. This is an upper limit and shows explicitly that the uncertainty is dominated by systematics.

\section{The future of GC research: GRAVITY}

Given certain orbital elements for an S2-like star, the deviations between a Keplerian orbit and a fully relativistic orbit are on the order of 10 mas. However, the orbital elements are not known a priori but are determined from the same fit. Thus, one has to ask a question how much of the deviation will be there between the models for a certain data set. The result is a smaller number since the orbital elements can absorb much of the changes due to relativity. An explicit simulation shows that the remaining deviations are typically $100 \mu \mathrm{as}$, which unfortunately is beyond what is feasible today.

If one could observe test particles closer to the point mass, the detection of postNewtonian effects would become easier. The density profile of the central star cusp as obtained from number counts (Genzel et al. 2003, Schödel et al. 2007) predicts that in the central 100 mas there should reside a few $(\sim 5)$ stars with K-band magnitudes from 
17 to 19 . Such stars would have orbital periods of roughly 1 year and the relativistic precession will be a few degrees per revolution.

In order to spatially resolve such stars one needs to increase the angular resolution of the instrument beyond the diffraction limit of existing large telescopes with diameters of $8-10 \mathrm{~m}$. The highest angular resolution will be possible using interferometry, since baselines of $\sim 100 \mathrm{~m}$ are envisaged, beating even the currently planned extremely large telescopes which will have apertures of $30-40 \mathrm{~m}$. The faintness of the sources aimed at essentially dictates the use of large telescopes for the interferometer. The only observatory that will allow the combination of several $8 \mathrm{~m}$ class telescopes is the ESO VLTI. The angular resolution achievable is 1.5 mas, the astrometric accuracy should be around $10 \mu$ as.

Since both an extended mass distribution and the relativistic effects will influence the shapes of future interferometric orbits in the GC, one actually needs at least two stars to disentangle the two effects. We have simulated the interferometric field of view of VLTI and could show that it is feasible to detect many stars in the field. Since the u-v-plane cannot be sampled very densely due to the limited number of baselines, the effective point spread function of the VLTI is a relatively complicated beam. Therefore one needs deconvolution techniques similar to what was developed for radio interferometry.

Probably the most exciting application of $10 \mu$ as astrometry in the GC is the observation of flares from Sgr A*, sporadic emission events that last one to two hours and for which it is known that they happen very close to the event horizon that has an apparent size of $10 \mu \mathrm{as}$. Therefore the ability to measure positions (and thus motions) with a similar accuracy opens a new window for testing gravitational theories in a very strong gravitational field. The apparent path of an assumed plasma hot spot orbiting the black hole is suspect to many relativistic effects, such as gravitational redshift, Doppler boosting and multiple images (Broderick \& Loeb 2006). Therefore the GC offers the unique chance to directly observe the effects of strong gravity.

Current instrumentation at the VLTI misses the goal to observe the GC. We have identified the following key features needed for the GC experiment as described:

- Combining the light of four $8 \mathrm{~m}$ telescopes

- NIR wavefront sensing for the AO of each telescope.

- Fringe tracking on a nearby field star with $m_{K}=10$.

Recently we have finished a feasibility study for an interferometric instrument combining these features, called "GRAVITY". Besides a compelling science case we could show that the instrument is technically feasible and that the GC field offers bright enough field stars for both AO and fringe tracking. The concept for the instrument is presented in poster 2.2.3 of this IAU workshop (Eisenhauer et al. 2008).

\section{References}

Broderick, A. \& Loeb, A. 2006, ApJ 636, 109

Eisenhauer, F. et al. 2005, ApJ 628, 246

Eisenhauer, F. et al. 2008, Proc. IAU 248, in this volume p.100

Genzel, R. et al. 2003, ApJ 594, 812

Ghez, A. et al. 2005, ApJ 620, 744

Morris, M. 2003, ApJ 408, 496

Muno, M. 2005, ApJ 622, L113

Reid, M. et al. 2007, ApJ 659, 378

Schödel, R. et al. 2002, Nature 419, 694

Schödel, R. et al. 2007, A\&A 465, 125 